\title{
An approach towards development of evaluation framework for usability of smartphone applications
}

\begin{abstract}
As software and web applications run on the same machine, traditional usability evaluation techniques can be easily conducted for these applications. Whereas, mobile application operates on profoundly different machine and environment; smartphones. The unique characteristics of mobile phones pose challenges in adopting traditional usability evaluation techniques for evaluating usability of mobile applications. This implies that mobile applications also have different properties of user interface compared to software and web application. Thus, adopting traditional usability evaluation methods for mobile applications are prone towards unreliable usability analysis. Previous studies proposed usability evaluation framework for native mobile phone functionalities or physical constraints, limitations and mobility conditions of mobile devices based on user interface widgets. This paper proposed a framework with an integrated approach to usability evaluation for a smartphone app in view of abstraction levels of usability criteria and user interface elements of an app.
\end{abstract}

Keyword: Mobile usability; Smartphone apps; Usability evaluation method; Usability framework 